\title{
A POISSON KERNEL FOR CERTAIN HOMOGENEOUS SPACES
}

\section{ROBERT HERMANN ${ }^{1}$}

1. Introduction. The aim of this note is to prove some of the results of D. Lowdenslager [6] on potential theory in symmetric, bounded domains in a more general setting. In particular, our methods are not restricted to complex manifolds nor to spaces imbeddable in Euclidean space.

The main result is Theorem 3.1 , which presents a Lie grouptheoretic method for constructing a Poisson kernel for certain homogeneous spaces $G / K$, with $G$ a noncompact Lie group, $K$ a compact, connected subgroup.

In section 2 we present several remarks that are meant to indicate how some of the results in [6] on the Bergman-Šilov boundary of a Cartan domain can be adapted to other situations, namely when a homogeneous space is equivariantly imbedded in a bigger space. Unfortunately, we do not yet know of any examples other than the Cartan domains where all of this machinery can be applied, although there are indications of this possibility in Karpelevič's work [5]. However, it is at least evident that results such as these that are not so dependent on explicit calculation as Lowdenslager's [6] are useful in dealing with the exceptional Cartan domains.

2. The Bergman-Šilov boundary. All manifolds, Lie groups, action of Lie groups on manifolds, tensor-fields, maps, etc. will be of differentiability class $C^{\infty}$ unless mentioned otherwise. We follow Chevalley [3] for Lie group and differential-geometric notations, with some of the modifications suggested in [1]. All manifolds will be assumed to be connected and paracompact. Let $M$ be such a manifold. For $x \in M, M_{x}$ is the tangent space to $M$ at $x$. If $\phi: M \rightarrow M^{\prime}$ is a map of manifolds, $\phi_{*}: M_{x} \rightarrow M_{\phi(x)}^{\prime}$ denotes the linear map that $\phi$ indicates on tangent vectors. If $\omega$ is a differential form on $M^{\prime}, \phi^{*}\left(\omega^{\prime}\right)$ denotes the form on $M$ induced by the map $\phi$.

Let $G$ be a Lie group that acts on $M$. This means that, for each $g \in G$, there is a diffeomorphism $T_{g}: M \rightarrow M$ such that:

(a) $T_{o_{102}}=T_{o_{1}} T_{o_{2}}$ for $g_{1}, g_{2} \in G$.

(b) $T_{\bullet}=$ identity map of $M$, where $e$ is the identity element of $G$. We write:

$T_{o}(x)=g x$ or $g \cdot x$ for $g \in G, x \in M$. Let $G$ be the Lie algebra of $G$.

Received by the editors November 28, 1960.

${ }^{1}$ Lincoln Laboratory, Massachusetts Institute of Technology, operated with support from the U.S. Army, Navy and Air Force. 
Let $V(M)$ be the set of vector fields on $M$, considered as an infinite dimensional Lie algebra with respect to the Jacobi bracket operation.

If $X \in V(M)$, and $\omega$ is a differential form on $M$, let $X(\omega)$ denote the Lie derivative of $\omega$ by the vector field $X$. (This is denoted by $\theta(X)(\omega)$ in [2], to which we also refer for the definition of this operation.)

Let $C^{\infty}(M)$ be the ring $C^{\infty}$ real-valued functions on $M$. The operation of Lie derivation with respect to the vector field $X$ on $M$ defines a derivation of $C^{\infty}(M)$, i.e., $X$ defines a first-order, linear differential operator on $M$.

More or less as definition of the Jacobi-bracket operation, we have:

$$
[X, Y](f)=X(Y(f))-Y(X(f)) \text { for } X, Y \in V(M), f \in C^{\infty}(M) \text {. }
$$

The action of $G$ on $M$ defines a Lie-algebra homomorphism $\rho: G \rightarrow V(M)$. As definition:

For $X \in G, x \in M, \rho(X)(x)$ is the tangent vector to the curve $t \rightarrow \operatorname{Exp}(t X) x$ at $t=0$. One shows that:

(2.1) For $g \in G, X \in G, f \in C^{\infty}(M), T_{g}^{*}(\rho(X)(f))=\rho(\operatorname{Ad} g(X)) T_{\theta}^{*}(f)$.

For $x \in M$, let $G x$ be the orbit of $G$ at $x, L_{x}$ be the isotropy group of $G$ at $x . G x$ is isomorphic in a natural way to $G / L_{x}$, the space of right cosets.

If $S$ is a subset of $M$ such that $G \cdot S \subset S$, define $C^{\infty}(S)$ (resp. $C^{\infty}(S)$ ) as the ring of all real valued, continuous (resp. not necessarily continuous) functions that are $C^{\infty}$ when restricted to the orbits of $G$ on $S$.

We suppose that $G$ has a nondegenerate quadratic form $($,$) .$ that is invariant under $\operatorname{Ad} G$. Choose a basis for $G$,

$\left(X_{i}\right), 1 \leqq i \leqq n$, such that

Set $A_{i}=\left(X_{i}, X_{i}\right)$. Define the second-order linear differential operation $\Delta$ on $C^{\infty}(M)$ as follows:

$$
\Delta(f)=\sum_{i=1}^{n} A_{i} \rho\left(X_{i}\right) \rho\left(X_{i}\right)(f), \quad \text { for } f \in C^{\infty}(M) .
$$

It is clear that it is independent of the basis chosen for $G$ satisfying 2.2. Also, note that 2.1 implies that $\Delta$ is invariant under the action of $G$, i.e.,

Note that:

$$
T_{o}^{*}(\Delta(f))=\Delta T_{0}^{*}(f) \quad \text { for } f \in C^{\infty}(M), G \in G .
$$

For $X \in G, f \in C^{\infty}(M), \rho(X)(\rho(X)(f))(x)=\left.\frac{\partial^{2}}{\partial t^{2}} f(\operatorname{Exp}(X t) \cdot x)\right|_{t=0}$. 
In particular, if $X \in L_{x}$, the Lie algebra of the isotropy group at $x$, then $\rho(X)(\rho(X)(f))(x)=0$.

Suppose that the basis $\left(X_{i}\right)$ is chosen so that $X_{i}, 1 \leqq i \leqq m$ spans $\boldsymbol{L}_{x}$, and $X_{i}, m+1 \leqq i \leqq n$ spans $\boldsymbol{M}_{x}$, the orthogonal complement of $L_{x}$ with respect to $($,$) . Then,$

$$
\Delta(f)(x)=\sum_{i=m+1}^{n} A_{i} \rho\left(X_{i}\right)\left(\rho\left(X_{i}\right)(f)\right)(x) .
$$

As an application of these remarks, suppose that the form $($, restricted to each $\boldsymbol{M}_{x}$ is positive definite and $G$ is transitive on $M$, i.e., the map $X \rightarrow \rho(X)(x)$ of $G \rightarrow M_{x}$ is onto with kernel $L_{x}$. This defines an isomorphism of $M_{x}$ with $\boldsymbol{M}_{\boldsymbol{x}}$, hence a positive-definite quadratic form on $M_{x}$, i.e., $M$ is a Riemannian manifold, with $G$ acting as a group of isometries. It is known [7] that, for each $X \in \boldsymbol{M}_{x}$, the curve $t \rightarrow \operatorname{Exp}(t X) \cdot x$ is a geodesic. Then 2.4, together with the classical formula for the Laplacian operator in Riemann normal coordinates, prove the following result:

Proposition 2.1. With the assumptions described above, $\Delta$ is the Laplace operator of the Riemannian metric on $M$ invariant under $G$.

We return now to the case where $G$ is not necessarily transitive on $M$. Let $S$ be a subset of $M$ such that $G \cdot S \subset S$. Each $X \in G$ defines a derivation of $C^{\infty}(S)$ that, since $\rho(X)$ is tangent to $S$, is compatible with the derivation $\rho(X): C^{\infty}(M) \rightarrow C^{\infty}(M)$ and the restriction mapping: $C^{\infty}(M) \rightarrow C^{\infty}(S)$. Formula 2.3 then defines, for $f \in C^{\infty}(S)$, a differential operator

$$
\Delta_{S}: C^{\infty}(S) \rightarrow C^{\infty}(S) .
$$

It is clear that this is equivalent to saying that the operator $\Delta$, when expressed in local coordinates about a point of $S$, has no component normal to $S$.

Now, fix a point $x_{0} \in M$. Let $D=G \cdot x_{0}, L=L_{x_{0}}, \boldsymbol{M}=\boldsymbol{M}_{x_{0}}, L=\boldsymbol{K}_{x_{0}}$. Let $\bar{D}$ be the closure of $D$ in $M$, and let $F=\bar{D}-D$ be its boundary. $\bar{D}$ and $F$ are invariant under the action of $G$. Suppose the basis $\left(X_{i}\right)$ of $G$ is chosen to satisfy 2.2 and so that $\left(X_{i}\right), 1 \leqq i \leqq m$, is a basis for $L$, $\left(X_{i}\right), m+1 \leqq i \leqq n$, is a basis for $\boldsymbol{M}$, and, for all such $i,\left(X_{i}, X_{i}\right)$ $=A_{i}>0$. Then, we see from 2.4 that:

$\Delta_{D}$ is a positive definite, second-order differential operator on the manifold $D . \Delta_{F}$ is a positive semi-definite operator on $F$.

Definition 2.1. The Bergman-Silov boundary of $D$ is the set $B$ of $x \in F$ such that: $\Delta_{F}(f)(x)=0$ for all $f \in C^{\infty}(F)$, i.e. $B$ is the set of points where the coefficients of the operator $\Delta_{F}$ vanish identically. 
It is clear from Lowdenslager's work [6] that $B$ defined in this grouptheoretic way has a close relation with the points of $F$ at which functions in $C^{\infty}(\bar{D})$ that are harmonic with respect to $\Delta_{D}$ take their maximum value. Along this line, we now prove, using the same methods, the following generalizations of Lemma 1 of [6]:

Proposition 2.2. Suppose that $f \in C^{\infty}(\bar{D})$ satisfies: $\Delta(f) \geqq 0$ and that $F$ is compact.

(a) If there is an $\omega \in C^{\infty}(\bar{D})$ such that $\Delta(\omega)(x)>0$ for all $x \in \bar{D}-B$, then $f$ must take its maximum value at a point of $B$.

(b) If $f$ has a strong relative maximum at $x \in F$, then $x \in B$.

Proof. If (a) were not true, there would be an $\epsilon>0$ such that $f+\epsilon \omega$ takes a maximum value outside $B$. But, $\Delta(f+\epsilon \omega)>0$ outside $B$, and $\Delta=\Delta_{F}$ on $F$, which contradicts the fact that the Hessian of $f+\epsilon \omega$ must be negative semi-definite at some point outside $B$.

Part (b) follows from the same argument on noting that such an $\omega$ can always be chosen locally about $x$ if $x \in B: f+\epsilon \omega$ can be made to have a maximum value at a point arbitrarily close to $x$ by choosing $\epsilon$ sufficiently small, since $f$ takes a strong relative maximum at $x$. This again is a contradiction.

Recall that in Lowdenslager's situation, where $M$ is Euclidean space, $\omega$ can be taken as a convex function.

3. The Poisson kernel. From now on, suppose that $D$ and $B$ are $C^{\infty}$ manifolds on which a connected Lie group $G$ acts. We do not assume that $B$ lies on the boundary of $D$ in any sense. We assume further that $G$ has a quadratic form invariant under $\operatorname{Ad} G$ that enables us to define invariant second-order linear operators on $B$ and $D, \Delta_{B}$ and $\Delta_{D}$. The problem to be discussed deals with conditions that at least some of the functions $f \in C^{\infty}(D)$ harmonic with respect to $\Delta_{D}$ can be expressed as integrals with respect to a "Poisson kernel" of functions on $B$.

Fix a point $x_{0} \in D$ and let $L\left(=L_{x_{0}}\right)$ be the isotropy group of $G$ at $x_{0}$. We suppose that:

(3.1) $B$ has a $C^{\infty}$ measure $d b$ (i.e., an everywhere nonzero differential form of maximal dimension) which is invariant under the action of $K$ on $B$.

For $g \in G$ (resp. $X \in G$ ) let $J_{g}$ (resp. $J_{X}$ ) be the Jacobian of $T_{\theta}$ (resp. $\rho(X)$ ) with respect to $d b$, i.e.

$$
\begin{aligned}
T_{o}^{*}(d b) & =J_{o} d b, \\
\rho(X)(d b) & =J_{X} d b .
\end{aligned}
$$


(Recall that $\rho(X)$ denotes the operation of Lie derivation with respect to the vector-field on $B$ defined by $X \in G$.)

We see from 3.2 and 3.3 that:

$$
\begin{aligned}
J_{\theta_{1} g_{2}} & =T_{g_{2}}^{*}\left(J_{\theta_{1}}\right) J_{g_{2}} & \text { for } g, g_{2} \in G . \\
J_{[X, Y]} & =\rho(X)\left(J_{Y}\right)-\rho(Y)\left(J_{X}\right) & \text { for } X, Y \in G . \\
J_{\mathbf{A d} g(X)} & =T_{g}^{*}\left(J_{X}\right) J_{g} & \text { for } g \in G, X \in G .
\end{aligned}
$$

A function $K(x, b) \in C^{\infty}(D, B)$ is said to be an equivariant kernel for $D \times B$ providing:

$$
K(g x, b)=K\left(x, g^{-1} b\right) J_{g^{-1}}(b) \quad \text { for all } g \in G, b \in B, x \in D .
$$

Let $C_{0}^{\infty}(B)$ be the subring of $C^{\infty}(B)$ of functions with compact support. A kernel $K(x, b)$ satisfying 3.7 defines a linear mapping $K: C_{0}^{\infty}(B)$ $\rightarrow C^{\infty}(D)$ as follows:

$$
K(f)(x)=\int K(x, b) f(b) d b \quad \text { for } f \in C_{0}^{\infty}(B) .
$$

Note that:

$$
T_{o}^{*}(K(f))=K\left(T_{0}^{*}(f)\right) \quad \text { for } f \in C_{0}^{\infty}(B), g \in G .
$$

Differentiating 3.7 with respect to $g$, we have:

$$
\rho(X)_{x}(K(x, b))=-\rho(X)_{b}(K(x, b))-K(x, b) J_{X}(b)
$$

for all $X \in G, x \in D, b \in B$.

$\left(\rho(X)_{x}\right.$ and $\rho(X)_{b}$ denote partial Lie derivation with respect to the vector field defined by $X$ on the $x$-space (i.e., $D$ ) and the $b$-space (i.e., $B$ ).) Applying $\rho(X)_{x}$ to 3.10 on both sides, and using 3.10 again, we have:

$$
\begin{aligned}
\rho(X)_{x}\left(\rho(X)_{x}(K(x, b))\right) & \\
= & \rho(X)_{b}\left(\rho(X)_{b}(K(x, b))\right)+2 \rho(X)_{b}(K(x, b)) J_{X}(b) \\
& +K(x, b)_{\rho}(X)_{b}\left(J_{X}(b)\right)+K(x, b)\left(J_{X}(b)\right)^{2} .
\end{aligned}
$$

For $X \in G, x \in D$, define $F_{X, x} \in C^{\infty}(B)$ by:

(3.12) $\quad F_{X, x}(b)=\left[2 \rho(X)_{b}(K) J_{X}+K \rho(X)_{b}\left(J_{X}\right)+K J_{X}^{\&}\right](x, b)$,

i.e. everything but the first term on the right-hand side of 3.11. This is the "obstruction" to changing $\rho(X)_{x} \rho(X)_{x}$ into $\rho(X)_{b} \rho(X)_{b}$.

$$
T_{k}^{*}\left(F_{X, x}\right)=F_{\mathrm{Adk}(X), k-1_{x}} \text {, using 2.1, 3.6 and 3.7. }
$$

We are now ready to state the main result: 
THEOREM 3.1. Suppose that, in addition to the conditions stated above,

(a) $L$ is transitive on $B$ and $G$ is transitive on $D$,

(b) $\Delta_{B}$ is identically zero,

(c) $K(x, b)$ satisfies 3.7 and

(d) $\int K(x, b) d b=1$ for all $x \in D$.

Then $K(x, b)$ is, for each $b$ held fixed, a solution of the equation $\Delta_{D}=0$. In particular, $K$ maps all of $C_{0}^{\infty}(B)$ into solutions of the differential equation: $\Delta_{D}=0$.

Proof. With condition (b) we have, by 3.11 ,

$$
\Delta_{D} K(x, b)=\sum_{i=1}^{n} A_{i} F_{X_{i}, x}(b) \text {. }
$$

Conditions (b) and (d) imply that

$$
\int \sum_{i=1}^{n} A_{i} F_{X_{i}, x}(b) d b=0 .
$$

Notice, however, that for $k \in K$,

$$
\sum_{i=1} A_{i} F_{\mathrm{Ad} k\left(X_{i}\right), x_{0}}(k b)=\sum_{i=1} A_{i} F_{X_{i}, x_{0}}(b) .
$$

Referring to $3.12,3.6$, and 2.1 , we see that:

$$
\sum_{i=1}^{n} A_{i} F_{\mathrm{Ad} k\left(X_{i}\right), x_{0}}=\sum_{i=1}^{n} A_{i} F_{X_{i}, x_{0}}
$$

i.e., using 3.16 and condition (a), the integrand in 3.15 is constant. Hence:

$$
\sum_{i=1}^{n} A_{i} F_{X_{i}, x_{0}}(b)=0 \quad \text { for all } b \in B .
$$

For each $b \in B$, let $K_{b} \in C^{\infty}(D)$ be the function:

$$
K_{b}(x)=K(x, b) \quad \text { for } x \in D .
$$

Then, by 3.14 and 3.18 ,

$$
\Delta_{D}\left(K_{b}\right)\left(x_{0}\right)=0 \quad \text { for all } b \in B .
$$

To finish the proof, we must show that this holds for all points of $D$. Suppose that $x=g \cdot x_{0} \in D$ for some $g \in G$.

$$
\begin{aligned}
\Delta_{D}\left(K_{b}\right)(x) & =T_{0}^{*}\left(\Delta_{D} K_{b}\right)\left(x_{0}\right), \\
& =\Delta_{D}\left(T_{0}^{*}\left(K_{b}\right)\right)\left(x_{0}\right), \text { using invariance of } \Delta_{D} \text { under } G, \\
& =\Delta_{D}\left(K_{\theta^{-1} b} J_{\theta^{-1}}(b)\right)\left(x_{0}\right), \text { by } 3.7, \\
& =\Delta_{D}\left(K_{\theta^{-1} b}\right)\left(x_{0}\right) J_{\theta^{-1}}(b)=0, \text { by } 3.19 .
\end{aligned}
$$


This completes the proof of Theorem 3.1.

Proposition 3.2. Suppose that $G$ acts on manifolds $D$ and $B$, that $B$ is compact, $L \subset G$ is the isotropy sub group of $G$ at $x_{0} \in D$, that $K$ is transitive on $B$, and that the isotropy subgroup $L_{x}$ of $G$ at each $x \in D$ is compact.

Then, there is a $C^{\infty}$ measure $d b$ on $B$ and a function $K(x, b) \in C^{\infty}(D, B)$ satisfying 3.7 and the condition (d) of Theorem 3.1.

Proof. Since $L_{x}$ and $B$ are compact, there is a $C^{\infty}$ measure $d_{x} b$ on $B$ invariant under $L_{x}$ such that

$$
\int_{B} d_{x} b=1 \quad \text { for each } x \in D .
$$

Set: $d b=d_{x_{0}} b$, and $d_{x} b=K(x, b) d b$.

One sees easily that $K(x, b)$ satisfies the required conditions. Note also that:

$$
K(x, b)=J_{\theta^{-1}}(b) \text { if } x=g \cdot x_{0} .
$$

Remarks. (a) Boundary behavior. For $X \in G$, suppose that there is a subset $A \subset B$ of measure zero and a point $b_{0} \in B$ such that:

$$
\text { For all } b \in B-A, \quad \lim _{t \rightarrow \infty} \operatorname{Exp}(t X) \cdot b=b_{0} .
$$

Following Lowdenslager [6] we note then that:

$$
\lim _{t \rightarrow \infty} \int K\left(\operatorname{Exp}(t X) \cdot x_{0}, b\right) f(b) d b=f\left(b_{0}\right)\left(\text { use 3.20) for all } f \in C_{0}^{\infty}(B),\right.
$$

i.e. the function in $D$ defined by the Poisson formula has the correct boundary behavior along the ray starting from $x_{0}$ determined by $X$. One can prove that the following conditions imply 3.21 .

(3.23) (a) $B$ is compact.

(b) There is a Riemannian metric on $B$ and a $f \in C^{\infty}(B)$ such that $\rho(X)$ on $B$ is the gradient field of $f$.

(c) $f$ has only one relative maximum, at $b_{0}$.

(d) Let $N \subset B$ be any connected component of the set of other critical points of $f$. We require that there be a neighborhood $U$ of $N$ and a submanifold $S$ of $U$ of lower dimension containing $N$ such that, for $b \in U$, the curve $t \rightarrow \operatorname{Exp} t X \cdot b$ approaches $N$ as $t \rightarrow \infty$ if and only if $b \in S$. ( $S$ is a stable manifold for the vector field $\rho(X)$ near its critical set).

The point is that, at least for the case where $D$ is a Cartan domain 
and $M$ is its compactification, it can be shown that these conditions follow from rather general differential-geometric arguments. We will come back to this point of view in a later work.

(b) The general setting of Theorem 3.1. Theorem 3.1 involves a condition for commutativity of equivariant differential and integral operators. For other sufficient conditions for this sort of phenomenon, see [8].

(c) Application to the Cartan domains. Suppose that $D$ is a Cartan domain and that $M$ is the compact Kähler manifold in which $D$ is equivariantly embedded as an open set. Then, as above, $D=G / L$, where $G$ is an non-compact, simple Lie group, $L$ is a maximal connected compact subgroup of $G, M=G_{u} / L$, where $G_{u}$ is a compact real form of the complex simple Lie group determined by $G$. $G / L$ is then a Riemannian symmetric space, and $G$ admits a decomposition $\boldsymbol{L} \oplus \boldsymbol{M}$, with $[M, M] \subset \boldsymbol{L}$. Let $\boldsymbol{H}$ be a maximal abelian subalgebra of $M$. Let $W$ be a Weyl chamber of $H$. Define $B \subset \bar{D}-D$ as in definition 2.1. It follows from the results of [4] that $\lim _{t \rightarrow \infty} \operatorname{Exp}(X t) \cdot x_{0} \in B$ for $X \in W$, and for $X$ lying on the boundary of $W, \lim _{t \rightarrow \infty} \operatorname{Exp} X t \cdot x_{0} \notin B$. $\mathrm{R}$. Bott and A. Koranyi have shown (to appear) that there is a $b_{0} \in B$ such that: $\lim _{t \rightarrow \infty} \operatorname{Exp} X t \cdot x_{0}=b_{0}$ for all $X \in W$, and that $L$ acts transitively on the orbit of $G$ at $b_{0}$. (We can show this also with the geometric method described in remark (a) above.) It follows from these facts that $G$ and $L$ both act transitively on $B$.

All of these facts are due to D. Lowdenslager, ([6], together with some unpublished work) in the classical cases. However, it is useful for the sake of possible generalizations to have general proofs of these results.

\section{BiBLIOGRAPHY}

1. W. Ambrose and I. M. Singer, A theorem on holonomy, Trans. Amer. Math. Soc. vol. 75 (1953) pp. 428-443.

2. H. Cartan, Notions d'algèbre différentielle, Colloque de Topologie de Bruxelles, 1950, pp. 16-27.

3. C. Chevalley, Lie groups. I, Princeton, Princeton University Press, 1946.

4. R. Hermann, Geodesics of bounded, symmetric domains, Comment. Math. Helv. vol. 35 (1961) pp. 1-8.

5. F. I. Karpelevič, Geodesics and harmonic functions on symmetric spaces, Dokl. Akad. Nauk SSSR vol. 124 (1959) pp. 1199-1202. (Russian)

6. D. Lowdenslager, Potential theory in bounded, symmetric homogeneous complex domains, Ann. of Math. vol. 67 (1958) pp. 467-484.

7. K. Nomizu, Invariant affine connections on homogeneous spaces, Amer. J. Math. vol. 76 (1954) pp. 33-65.

8. A. Selberg, Harmonic analysis and discontinuous groups in weakly symmetric Riemannian spaces . . , J. J. Ind. Math. Soc. vol. 20 (1956) pp. 47-87.

Lincoln Laboratory, Massachusetts Institute of Technology 